\title{
Management of Surgical Procedures in Patients with Inherited F VII Deficiency: Six Years of Experience
}

\author{
Radomira Hrdlickova1, Sarka Blahutova ${ }^{1}$, Roman Hajek ${ }^{1,2}$ and Zuzana Cermakova ${ }^{1,2 *}$ \\ ${ }^{1}$ Haemophilia Treatment Centre, Department of Haematooncology, University Hospital Ostrava, Czech Republic \\ ${ }^{2}$ Faculty of Medicine, University of Ostrava, Czech Republic
}

\begin{abstract}
Inherited factor VII (FVII) deficiency is the most frequently observed rare bleeding disorder. The clinical symptoms are extremely variable, and patients may be asymptomatic or may present with life-threatening bleeding disorders. FVII activity (FVII: C) values of $10-15 \%$ are considered safe for maintenance of hemostasis, and replacement therapy is recommended for some types of surgical procedures. Twenty-three adult patients with FVII:C deficiency are followed-up in the Ostrava Haemophilia Treatment Centre. Eleven patients underwent a total of fourteen invasive procedures between 2008 and 2013. In terms of replacement therapy, nine patients received plasma-derived FVII and five patients received activated recombinant FVII. None of the patients had excessive blood loss during surgery, and there were no bleeding or other complications during post-operative treatment. In addition, there were no thromboembolic events related to the use of replacement therapy.
\end{abstract}

Keywords: Inherited FVII deficiency; Surgery; Replacement therapy; Antithrombotic prophylaxis

\section{Introduction}

Inherited coagulation factor VII deficiency is the most frequently observed rare inherited coagulation disorder. The heredity of the disease is autosomal recessive, and the prevalence of a serious disorder (FVII: $\mathrm{C}<2 \%$ ) is estimated to be 1:300,000-1:500,000 inhabitants [1]. FVII: $\mathrm{C}$ shows variable clinical manifestation and can range from a clinically asymptomatic course in which only laboratory findings are used for the diagnosis to moderate bleeding disorders to lifethreatening bleeding episodes [2,3]. In many cases, the seriousness of the bleeding does not correspond to the seriousness of the laboratory findings and most probably is not influenced by the genetic mutation either. For example, one individual with very low residual FVII activity may exhibit no bleeding problems or only a minor bleeding disorder, whereas another patient with much higher FVII activity may suffer from a serious bleeding disorder. Interestingly, patients with the exact same homozygous mutation can have different clinical phenotypes. It is thus likely that other genetic factors or environmental factors also play important roles in the disorder [3,4]. Clinical manifestations of FVII:C deficiency include predominant bleeding into tissues rich in tissue factor such as the brain, intestines, uterus, placenta, and lungs, and bleeding episode onset may be associated with a surgical procedure [2]. Frequent hemorrhages in women can result in irondeficiency anemia. Bleeding into joints and muscles is not as typical as in hemophilia; nevertheless, this can occur in both men and women with the disorder. Upon laboratory examination, FVII:C deficiency is characterized by isolated prolongation of the prothrombin time, which is not adjusted with a corrective test performed with normal plasma. Low levels of FVII:C can also be detected by determining the functional activity of FVII with the one-stage prothrombin test, which is based upon the principle of the prothrombin test with optical detection using FVII-deficient plasma. FVII: C values of $10-15 \%$ are considered a hemostatic minimum, and replacement of FVII is recommended for surgical procedures. Considering the safety risks, the replacement of choice is activated recombinant FVII (rFVIIa), or plasma-derived FVII concentrate (pdFVII); fibrin glue may also be used in certain circumstances to support local hemostasis (e.g. in stomatosurgery). When these preparations are not available, concentrate of prothrombin complex (PCC) factors or plasma may also be used [1].
The aim of study is to present a management of substitution therapy in patients with different level of fVII deficiency either with pdFVII or with rFVIIa in different invasive procedures performed within five years (2008 and 2013) at the University Hospital Ostrava, Czech Republic.

\section{Patients and Methods}

A total of 23 adult patients with FVII: C deficiency were treated and followed-up from 2008 to 2013 at the University Hospital Ostrava. The FVII: C level was below $1 \%$ in six patients, between 1.0 and $10.0 \%$ in ten patients, and between 10.1 and $35.0 \%$ in seven patients. The most frequently observed bleeding disorders in the female patients were frequent subcutaneous hematomas, epistaxis, and menorrhagia. The medical history of four patients included past episodes of serious bleeding and impaired wound healing following surgical procedures. We did not encounter any cases of intra-articular bleeding or bleeding into the gastrointestinal tract or the central nervous system. In eight of the patients, the diagnosis was made when preoperative coagulation tests were performed or during family screening. FVII activity was assessed with a coagulation test that used DG-FVII (Grifols) deficient plasma, the PT reagents TriniClot PT Excel S (Trinity Biotech), and the Sysmex CA 1500 analyzer.

We prepared and followed the perioperative courses of eleven patients who underwent fourteen surgical procedures between 2008 and 2013. Nine patients were treated with replacement therapy using pdFVII (Factor VII; Baxter Healthcare) (Table 1), and five were treated with rFVIIa (NovoSeven") (Table 2). Two of the surgical procedures were performed laparoscopically.

*Corresponding author: Zuzana Cermakova, Haemophilia Treatment Centre Department of Haematooncology, University Hospital Ostrava, Czech Republic, Tel: 420597371 111; E-mail: zuzana.cermakova@fno.cz

Received May 19, 2014; Accepted October 29, 2014; Published November 20, 2014

Citation: Hrdlickova R, Blahutova S, Hajek R, Cermakova Z (2014) Management of Surgical Procedures in Patients with Inherited F VII Deficiency: Six Years of Experience. J Blood Disorders Transf 5: 239. doi: 10.4172/2155-9864.1000239

Copyright: (C 2014 Hrdlickova R, et al. This is an open-access article distributed under the terms of the Creative Commons Attribution License, which permits unrestricted use, distribution, and reproduction in any medium, provided the original author and source are credited. 


\begin{tabular}{|c|c|c|c|c|c|c|c|c|c|c|}
\hline No. & Sex & Age, $y$ & FVII:C \% & Type of surgery & $\begin{array}{c}\text { pdFVII, } \\
\text { IU }\end{array}$ & $\begin{array}{c}\text { Body } \\
\text { weight, kg }\end{array}$ & IU/kg & Period of substitution & Bleeding & $\begin{array}{l}\text { Number } \\
\text { of IU/kg }\end{array}$ \\
\hline 1 & Female & 23 & 6.3 & Extraction of 3 retained molars & 1200 & 62 & 19 & $2 \times 600 \mathrm{IU}$ at $4 \mathrm{~h}$ & None; sutured & 19 \\
\hline 2 & Female & 44 & 6.5 & Molar extraction & 1200 & 65 & 18 & $2 \times 600 \mathrm{IU}$ at $4 \mathrm{~h}$ & None, sutured & 18 \\
\hline 3 & Male & 19 & 8.5 & $\begin{array}{c}\text { Subperiosteal abscess, molar } \\
\text { extraction }\end{array}$ & 1800 & 85 & 21 & $1200 \mathrm{IU}+600 \mathrm{IU}$ for $5 \mathrm{~h}$ & None, sutured & 21 \\
\hline 4 & Female & 39 & 4.0 & Mastopathy, excision of $3 \mathrm{~cm}$ nidus & 6000 & 97 & 62 & $48 \mathrm{~h}$ & Minimal & 62 \\
\hline 5 & Female & 45 & 5.0 & Reduction mammoplasty & 14,400 & 64 & 225 & 6 days & Minimal & 225 \\
\hline 6 & Male & 50 & 3.9 & Radical hernioplasty in cicatrix & 33,600 & 101 & 333 & 7 days & $\begin{array}{l}250 \mathrm{ml} \text { serosanguineous drainage } \\
\text { in the } 24 \mathrm{~h} \text { following surgery }\end{array}$ & 333 \\
\hline 7 & Male & 69 & 3.6 & Cataract surgery & 600 & 100 & 6 & $600 \mathrm{IU}$ prior to surgery & No bleeding & 6 \\
\hline 8 & Female & 47 & 0.5 & Hysteroscopy + abrasion & 3000 & 75 & 40 & $24 \mathrm{~h}$ & Minimal & 40 \\
\hline 9 & Male & 70 & 3.6 & Pacemaker reimplantation & 3600 & 100 & 36.00 & $24 \mathrm{~h}$ & Minimal & 36 \\
\hline
\end{tabular}

Table 1: Characteristics of patients with FVII: C deficiency who underwent surgery managed with pdFVII replacement therapy.

\begin{tabular}{|c|c|c|c|c|c|c|c|c|c|}
\hline No. & Patient & Age, y & FVII:C \% & Type of surgery & rFVIla, mg & $\begin{array}{c}\text { Body } \\
\text { weight, kg }\end{array}$ & ug/kg & $\begin{array}{l}\text { Period of } \\
\text { substitution }\end{array}$ & Bleeding \\
\hline 1 & Male & 63 & 1.4 & nephrectomy (clear-cell renal cell carcinoma) & 82 & 120 & 683 & 7 days & Minimal \\
\hline 2 & Female & 47 & 0.5 & cholecystectomy * & 64 & 74 & 865 & 7 days & Minimal \\
\hline 3 & Female & 49 & 4.6 & hernioplasty of recurrent umbilical hernia & 70 & 109 & 642 & 7 days & Minimal \\
\hline 4 & Female & 70 & 0.1 & $\begin{array}{c}\text { gynecological surgery (tumor of the minor pelvis } \\
\text { (fibroma on histology) }\end{array}$ & 67 & 80 & 838 & 7 days & Minimal \\
\hline 5 & Male & 52 & 3.9 & TEP of the hip joint & 81 & 97 & 835 & 10 days & $\begin{array}{l}<300 \mathrm{ml} \text { perioperatively } \\
+640 \mathrm{ml} \text { drainage } / 48 \mathrm{~h}\end{array}$ \\
\hline
\end{tabular}

Note: * laparoscopic surgery

Table 2: Characteristics of patients with FVII:C deficiency who underwent surgery managed with rFVII a replacement therapy.

\section{Results}

The FVII administration period for the eleven surgically treated patients varied from one to eight days; the exception was a patient who underwent Total hip replacement surgery (TEP) who received FVII for ten days. rFVIIa (15-30 ug/ $/ \mathrm{kg}$ ) was administered to five of the patients every four hours for the first two days and every six hours starting on the third day. Prothrombin time ratio (PT-R) was monitored every twelve hours on the day of surgery and once every 24 hours after surgery (prior to administration of the morning dose). In the other nine patients, the dose of pdFVII was calculated according to the following formula: required number of units = body weight $(\mathrm{kg})$ $\mathrm{x}$ required increase of FVII $(\%) \times 0.6$ [5]. This dose ensured that the plasma level of FVII:C at the time of surgery was around $20 \%$ and was maintained at $15-20 \%$ during wound healing following major surgical procedures. The FVII: C laboratory results were monitored every twelve hours on the day of surgery and once every 24 hours postoperatively except in patients receiving one-day replacement therapy. Except for patients undergoing cataract surgery, total hip replacement surgery, and renal tumor surgery, the patients were also given antifibrinolytic medication for wound healing for 7-10 days post-surgery (aminomethylbenzoic acid or tranexamic acid). Patients scheduled for major surgical procedures involving multiday hospitalization received prophylaxis for thromboembolic disorders with LMWH (low molecular weight heparin), used compression stockings, and underwent early rehabilitation.

\section{Discussion}

Inherited FVII: $\mathrm{C}$ deficiency is a relatively rare bleeding disorder. Nevertheless, it is likely that the indicated prevalence is underestimated or is regionally dependent, which was confirmed by the number of patients followed at our center (23 patients in a 6-year period).

In two of our female patients, the disorder was diagnosed based on preoperative assessment and was specifically diagnosed based on routine coagulation tests performed prior to planned hysterectomy.
The indication for the surgical procedure was reassessed following consultation with the attending gynecologist, and these two patients were treated with conservative therapy (anti-fibrinolytic medication plus hormonal therapy).

In making a differential diagnostics, it is important to differentiate between acquired FVII:C deficiency that occurs in the absence of vitamin $\mathrm{K}$ versus a clinically-mute history of bleeding disorders. The former is more likely in older patients, in neglected or oligophrenic patients with nutritional disorders, in patients with nutrition absorption problems, and in patients who have received long-term antibiotic therapy. The most frequent cause of acquired FVII:C deficiency is treatment with coumarins, which is usually apparent from the patient's pharmacological history.

Low levels of FVII:C do not protect the patient from possible thrombosis. In addition to classical cases of thromboembolic disorders, atypical thrombosis localization has been described. Thromboembolic disorders can also occur in very young patients and without any relation to replacement therapy, e.g. in the portal or splenic veins, central retinal vein, or axillar and cerebral veins [6]. That is why it is always necessary to carefully evaluate antithrombotic prophylaxis, especially in patients with multiday FVII replacement therapy.

\section{Conclusion}

We did not observe any excessive blood loss in the course of surgical procedures performed on patients treated with rFVIIa and pdFVII replacement therapy. None of the patients had bleeding complications during the postoperative course of treatment, and the surgical wounds healed per primam. None of the patients had thromboembolic complications. Thromboembolic prophylaxis was administered in one patient following total hip replacement for the recommended period of five weeks using LMWH without any hemorrhagic manifestations.

\section{Acknowledgement}

This work was supported by grants by MH CZ - DRO - FNOs/ 2012 and 
Citation: Hrdlickova R, Blahutova S, Hajek R, Cermakova Z (2014) Management of Surgical Procedures in Patients with Inherited F VII Deficiency: Six Years of Experience. J Blood Disorders Transf 5: 239. doi: 10.4172/2155-9864.1000239

Institutional development plan of University of Ostrava in 2012, financial resources are allocated by The Ministry of Education, Youth and Sports

\section{References}

1. Bolton-Maggs PH, Perry DJ, Chalmers EA, Parapia LA, Wilde JT, et al. (2004) The rare coagulation disorders--review with guidelines for management from the United Kingdom Haemophilia Centre Doctors' Organisation. Haemophilia 10: $593-628$

2. Lapecorella M, Mariani G; International Registry on Congenital Factor VII Deficiency (2008) Factor VII deficiency: defining the clinical picture and optimizing therapeutic options. Haemophilia 14: 1170-1175.
3. Mariani G, Bernardi F (2009) Factor VII Deficiency. Semin Thromb Hemost 35 400-406.

4. Peyvandi F, Di Michele D, Bolton-Maggs PH, Lee CA, Tripodi A, et al. (2012) Classification of rare bleeding disorders (RBDs) based on the association between coagulant factor activity and clinical bleeding severity. J Thromb Haemost 10: 1938-1943.

5. SPC Factor VII Baxter 600 IU.

6. Marty S, Barro C, Chatelain B, Fimbel B, Tribout B, et al. (2008) The paradoxical association between inherited factor VII deficiency and venous thrombosis. Haemophilia 14: 564-570. 